\title{
Airway clearance techniques for patients experiencing acute exacerbations of chronic obstructive pulmonary disease in the Republic of Ireland
}

\begin{abstract}
Hanrahan, C., Pedlow, K., \& Osadnik, C. (2021). Airway clearance techniques for patients experiencing acute exacerbations of chronic obstructive pulmonary disease in the Republic of Ireland. Physiotherapy Practice and Research, 42(2), 165-172. https://doi.org/10.3233/ppr-200499
\end{abstract}

Link to publication record in Ulster University Research Portal

Published in:

Physiotherapy Practice and Research

Publication Status:

Published (in print/issue): 10/12/2021

DOI:

10.3233/ppr-200499

Document Version

Author Accepted version

\section{General rights}

Copyright for the publications made accessible via Ulster University's Research Portal is retained by the author(s) and / or other copyright owners and it is a condition of accessing these publications that users recognise and abide by the legal requirements associated with these rights.

\section{Take down policy}

The Research Portal is Ulster University's institutional repository that provides access to Ulster's research outputs. Every effort has been made to ensure that content in the Research Portal does not infringe any person's rights, or applicable UK laws. If you discover content in the Research Portal that you believe breaches copyright or violates any law, please contact pure-support@ulster.ac.uk. 


\section{Title Page;}

2 Title of article; Airway clearance techniques for patients experiencing acute exacerbations of chronic obstructive pulmonary disease in the Republic of Ireland.

4 Authors names; Ciara Hanrahan ${ }^{1}$, Dr Katy Pedlow ${ }^{2}$, Dr Christian Osadnik ${ }^{3}$

1. Discipline of Physiotherapy, School of Clinical Therapies, Nano Nagle Place, Douglas Street, University College Cork, College of Medicine and Health Cork, Ireland T12X7OA

2. School of Health Sciences, Jordanstown Campus, Shore Road, Newtownabbey, Co. Antrim, BT37 OQB

3. Department of Physiotherapy, Monash University, Penninsula Campus, Building B, 47-49 Moorooduc Highway, Frankston, Victoria 3199, Australia.

Contact information for corresponding author; Ciara Hanrahan, Discipline of Physiotherapy, School of Clinical Therapies, Nano Nagle Place, Douglas Street, University College Cork, College of Medicine and Health Cork, Ireland T14X70A. Ciara.hanrahan@ucc.ie.Ph. 00353214904909

Wordcount; 2471 words 
Abstract:

Background and objectives: Airway clearance techniques (ACTs) are commonly prescribed for patients experiencing acute exacerbations of chronic obstructive pulmonary disease (AECOPD), however large variability in practice is commonly observed. This study aimed to explore current physiotherapy practice regarding ACTs for people with AECOPD in the Republic of Ireland.

Method: A cross-sectional online survey using multiple-choice questions and Likert scales was distributed to 202 physiotherapy clinicians via direct email and the Irish Society of Chartered Physiotherapists. Main survey themes included use of ACTs in relation to effectiveness and ease of use, and awareness of the literature and guidelines.

Results: Seventy responses (35\%) were received. The majority of respondents ( $n=$ $56,80 \%$ ) reported prescribing ACTs for more than $60 \%$ of patients with AECOPD with the most common techniques being physical activity $(n=65,93 \%)$ and active cycle of breathing techniques $(n=53,90 \%)$. Sputum management $(n=66,94 \%)$ was the most commonly reported indicator for use of ACTs. The majority of physiotherapists $(n=42,60 \%)$ reported being unsure of the literature or finding it conflicting regarding ACTs in AECOPD.

Conclusion: Physiotherapists in ROI regularly prescribe ACTs for patients with AECOPD despite a considerable reported lack of awareness of the evidence regarding their effectiveness. Further research is required to determine the reasons for this.

Key words; exacerbation, chronic obstructive pulmonary disease, sputum, airway clearance techniques, physiotherapist 
47 The authors have no conflict of interest to report.

48

49

50

51

52

53

54

55

56

57

58

59

60

61

62

63

64

65

66 


\section{Introduction and background;}

Chronic obstructive pulmonary disease (COPD) is a progressive, irreversible lung disease typically caused by exposure of the airways to noxious substances [1]. It is defined physiologically by a persistent obstructive airflow defect, and characterised by symptoms of breathlessness, cough and sputum production [1]. COPD is the third leading cause of death in the world and accounts for $6 \%$ of deaths internationally [2]. It is speculated almost 500,000 people ( $10 \%$ of the population) in the Republic of Ireland (ROI) could have COPD, with almost half of that figure likely to have moderate to advanced disease [3]. Acute exacerbations of COPD (AECOPD) involve acute deterioration of respiratory symptoms beyond normal day-to-day variability that require supplemental therapy [2]. Signs of AECOPD may include productive cough, increased sputum production, change in sputum colour and increased breathlessness [4].

In healthy lungs, secretions are cleared via cephalad transportation of mucus by cilia in the lining of the airways. This is recognised as an innate immuno-defence mechanism to protect against adverse effects of foreign particles [6]. In COPD, however, excess sputum and airways secretions are pathological disease components [7] that negatively impact upon this process, with cough and sputum linked to increased frequency of exacerbations and hospitalisation [5]. The presence of an abnormal defence mechanism to protect against excessive airway secretions justifies a role for supportive management strategies that aim to promote secretion clearance. Airway clearance techniques (ACTs) are techniques that aim to clear secretions via use of gravity, breath holding, forced expiratory technique and/or positioning, leveraging on key principles of alveolar interdependence and collateral ventilation [8]. Alveolar interdependence allows air to move into the smaller airways 
while expanding alveoli exert traction to surrounding poorly inflated alveoli [8]. When peripheral airways are obstructed, collateral ventilation provides alternative routes (in the form of channels) for air to flow [9]. In the literature, ACTs have been proposed to improve airflow to collateral channels and influence alveolar interdependence [9], however supportive evidence has proven challenging to emerge [10].

Evidence of clinical effectiveness regarding ACTs for people with AECOPD has existed for some time now, however conclusions in relation to the most effective ACTs are variable [11]. This is likely due to considerable heterogeneity of review methodologies and highly variable quality of individual clinical trials $[7,10,12,13]$. A small number of studies have explored physiotherapy practice of ACT prescription for the management of patients with AECOPD [15-19], demonstrating large variability in practice. For example, the most prescribed techniques in Australia were ACBT and physical exercise [18], while Swedish physiotherapists reported using PEP masks most [19]. No data exists relating to ROI.

In the ROI, use of ACTs by physiotherapists for COPD is outlined by the Health Services Executive (HSE) "End to End COPD Model of Care" clinical guidelines, which are based on the Global Initiative for Obstructive Lung Disease (GOLD) Guidelines [2, 3]. The HSE recommends use of ACTs by physiotherapists treating patients with COPD in the integrated care, acute hospital and COPD outreach settings but does not specify which ACTs are most effective or how to prescribe them.

The primary aim of the study was to determine current practice of physiotherapists with respect to ACTs and AECOPD in the ROI. Secondary aims were to explore perceptions of importance and effectiveness of ACTs, to identify factors influencing 
the prescription of ACTs and to examine physiotherapists awareness of evidence for ACTs in their management of AECOPD.

\section{Methods}

\section{Procedures}

A cross-sectional survey of physiotherapists treating patients with AECOPD in the ROI was implemented during January and February of 2020. The survey was conducted electronically via Survey Monkey due to clinicians' familiarity with the approach and to facilitate timely completion. Physiotherapists working in the ROI in the public or private health system were eligible for inclusion if they were registered with (or pending registration with) the national professional body CORU, and had treated patients with AECOPD within the last 10 years. For the purpose of this study an AECOPD was defined as an acute deterioration of a patients' respiratory symptoms requiring supplemental therapy [2], either in the community or following hospital admission. ACTs were defined as techniques used by a physiotherapist for the purpose of clearing sputum from patients' airways [18].

The survey comprised multiple-choice questions and Likert scales, based upon a previously used instrument $[18,19]$, with alteration of one question and addition of one other. The survey was reviewed by a respiratory clinical nurse specialist, a senior physiotherapist and a respiratory consultant physician in ROI to verify questions were appropriate for target respondents. Permission was granted to distribute the survey via email link to members of the Chartered Physiotherapists in Respiratory Care group (90 physiotherapists) and Chartered Physiotherapists Manager group (88 physiotherapy managers) of the Irish Society of Chartered 
140 Physiotherapists (professional body of the ROI). The survey was also emailed to physiotherapists throughout the ROI working in COPD outreach and respiratory integrated care services (approximately 24 physiotherapists), with all recipients encouraged to share the survey with colleagues experienced in respiratory care. In total, the approximate number of survey distributions was 202. To facilitate survey engagement, potential participants were emailed one week prior to initial survey distribution and reminders sent one, two and three weeks afterwards. Survey responses were also anonymised and data pertaining to work location and education history were not recorded. Ethical approval for the study was granted by Ulster University Ethics Board in December 2019.

Data analysis

Data were presented descriptively using frequencies, and percentages. Some data were pooled into fewer categories in order to explore relationships between variables. For example, work experience in the cardiorespiratory field was subdivided as $\leq 5$ vs 5 or more years, and similar approaches used for perceived effectiveness and awareness of the evidence regarding ACTs. Statistical significance was set at $p \leq 0.05$. Analyses were conducted using IBM SPSS statistical package version 26 and results presented as column graphs via Microsoft Excel.

\section{Results}

Participants

Responses were received from 70/202 (35\% response rate) physiotherapists who self-reported meeting study inclusion criteria. The majority of respondents $(n=59$, $84 \%)$ reported greater than 5 years experience, with $63 \%(n=44)$ working in cardiorespiratory physiotherapy for at least 10 years. As survey responses were 
anonymised, data pertaining to workplace (public, private, community or hospital based) were not included.

\section{Current practice}

167 The majority of respondents $(n=56,80 \%)$ reported prescribing ACTs for more than $60 \%$ of patients with AECOPD with only $7 \%(n=5)$ doing so rarely or never. The number of years working in cardiorespiratory did not appear to influence frequency of prescription of ACTs for patients with AECOPD $(p=0.65)$. The majority of respondents $(n=65,92 \%)$ spent between $5-20$ minutes on ACT treatment, with $3 \%$ $(n=2)$ spending 0-5 minutes, 40\% $(n=28)$ spending 5-10 minutes, 31\% $(n=22)$ spending $10-15$ minutes and $21 \%(n=15)$ spending $15-20$ minutes. $96 \%(n=69)$ of respondents felt that ACTs were important (very important, fairly important or

Perceptions

Physiotherapists perceived the most effective techniques for clearing sputum (very effective/ effective) to be physical activity ( $n=66,94 \%$ ), followed by ACBT ( $n=63$, $90 \%)$, oscillating PEP $(n=62,89 \%)$ and directed huffing $(n=60,86 \%)$. With respect to patient mastery of ACTs, respondents perceived physical activity ( $n=61,87 \%$ ), deep breathing ( $n=61,86 \%)$ and ACBT ( $n=52,74 \%)$ to be easiest (very easy/easy). There was no statistically significant relationship between perceived importance of ACTs and years of experience working in cardiorespiratory physiotherapy $(p=$ 
187

188

189

190

0.439). Frequency of use, perceived effectiveness and perceived ease of patients' mastery of airway clearance techniques is summarised in Figure 1.

\section{Clinical reasoning}

The most frequently identified indicators for prescribing ACTs were "difficulty managing secretions" ( $n=67,96 \%)$ and "recent change in sputum characteristics" $(n=63,90 \%)$ (Figure 2). The vast majority of respondents reported their primary aim of prescribing ACTs was "to clear sputum" (n=68, 97\%), "to improve oxygenation" $(n=59,84 \%)$, "enhance recovery from AECOPD" ( $n=57,81 \%)$ and "improve quality of life" ( $n=53,76 \%)$. Physiotherapists' reasoning for choosing between different ACTs was made on the basis of those deemed "easiest to master" $(n=51,73 \%)$, followed by "access to resources/equipment" ( $n=45,64 \%)$, "degree of dyspnoea" $(n=42,60 \%)$ and "patient preference" (41/70, 59\%).

\section{Knowledge of literature and guidelines}

While $40 \%$ ( $n=28)$ of respondents believed there was evidence supporting the use of ACTs during AECOPD, 40\% ( $n=28)$ believed the evidence was "conflicting, inconclusive or non-existent to support or refute use of ACTs". Twelve respondents (20\%) declared feeling "unsure what the current evidence is". Of the $80 \%$ of respondents who felt they were familiar with the literature, no differences were evident in terms of the extent of their experience between those working in cardiorespiratory more or less than 5 years and their awareness of the evidence for the effectiveness for ACTs with AECOPD ( $p=0.534)$. There was also no significant relationship between respondents who felt ACTs were important in the management of patients with AECOPD and perception of the strength of evidence to support the practice of ACTs for patients with AECOPD ( $p=0.377)$. Fifty $(71 \%)$ respondents 
indicated they were not familiar with Irish guidelines for the use of ACTs for patients with AECOPD.

\section{Discussion}

This was the first study of its kind to examine current practice of physiotherapists in ROI with respect to ACTs for patients with AECOPD. Results indicate ACTs are commonly used, and physical activity is perceived to be the most effective ACT. There appears to be a high degree of uncertainty, however, regarding the evidence to support this practice and poor awareness of existing national guidelines to support clinicians regarding this area of practice.

Treatment session duration (5-20 minutes) among physiotherapists from ROI appears consistent with previous reports $[18,19]$, however the high rate of ACT prescription (93\%) was greater than data from Australia (65\%) [18] and Sweden (75\%) [19]. This is despite similarities in the reported prevalence of COPD between countries. While the relationship between rate of prescription of ACTs and respondents perceived importance of them was not statistically significant in this study, an interesting observation between studies using this survey instrument has been the relationship between perceived importance of ACTs and years working in cardiorespiratory physiotherapy. As in this study, the Westerdahl et al study [19] did not report any significant differences in the frequency of prescription of ACTs and respondents working in cardiorespiratory more or less than 5 years. In Osadnik et als study [18], however, respondents with less than 5 years experience in cardiorespiratory reported prescribing ACTs more often than those with greater than 5 years experience $(p=0.017)$. This discrepancy may be reflected in undergraduate and postgraduate curricula, where more emphasis may be placed on teaching ACTs 
at undergraduate level in Australia. Further research would be warranted and may reveal some disparity in the delivery of such content internationally. Just under half of respondents believed there was evidence to support the use of ACTs during AECOPD. Yet, interestingly, a minority of respondents (36\%) reported they consider the evidence when choosing a suitable ACT. This finding has been previously observed $[18,19]$ and may reflect a degree of confusion interpreting the key messages from existing literature. It was concerning to observe the majority of respondents $(71 \%)$ were unaware of guidelines published by the Health Services Executive in $\mathrm{ROI}[3]$ in relation to the management of COPD, although these were only published one month prior to the survey. It would be valuable to examine the factors contributing to this observation.

While the use of physical activity as an ACT has not yet been directly examined in people with AECOPD, efficacy data is reported in people with stable cystic fibrosis. A single exercise session in a small adult CF sample $(n=14), F_{1} V_{1}$ range $19-108 \%$, was found to improve ease of sputum clearance [24] and a subsequent study by the same group [22] ( $n=24)$ found PEP and treadmill exercise augmented sputum clearance equally effectively. The mechanism proposed to explain these findings suggests PA increases peak expiratory flow and water content of viscous secretions, thus facilitating clearance. Differences between populations and pathophysiology of acute exacerbations, however, render it difficult to determine whether such findings would translate to people with AECOPD. Further enquiry into this area of practice would be of benefit to clinicians and patients, particularly in light of a recent study [26] that showed Australian physiotherapists frequently prescribe physical activity for patients with AECOPD, often in conjunction with huffing and coughing. This survey also showed referrals to pulmonary rehabilitation after discharge were very high, in- 
keeping with Australian guidelines [27] recommending that patients participate in pulmonary rehabilitation within two weeks of hospital discharge. Data suggests that pulmonary rehabilitation within 2 weeks after discharge from hospital with AECOPD can lead to rapid improvements in physical performance, when compared to delayed rehabilitation post COPD [28]. In ROI, The HSE Model of Care document on Pulmonary Rehabilitation [3] recommends referral within 3 weeks of hospital discharge, while The British Thoracic Society [28] advise referring patients on discharge from hospital and enrolling them in a pulmonary rehabilitation programme within one month. It would be interesting to speculate whether uptake of physical activity and/or pulmonary rehabilitation after AECOPD, whilst not typically implemented for the specific purpose of airway clearance, might accelerate recovery of issues related to excessive airway secretions. Furthermore, as 'ease of mastery' was cited as an important factor determining choice of specific ACT, it would be interesting to investigate the patient experience of performing ACTs, including physical activity, during AECOPD to reveal insights to further guide physiotherapy prescription.

\section{Limitations}

277 Findings from this survey need to be considered in light of some limitations. It is difficult to ascertain the precise number of people to whom this survey was distributed due to 'snowballing' recruitment methods and possible overlap of individuals across multiple dissemination sources and workplace settings. The low estimated response rate may mean insights lack some generalisability across broader ROI physiotherapy practice. It would be interesting to speculate whether a blended method of paper-based and online surveys, and a longer timeframe in which to conduct the study, might have optimised responses. As we did not record data of 
respondents' precise work locations, we were also unable to explore the potential for differences in ACT practice between physiotherapists working in outreach / integrated care sites and those based in hospitals. This would be useful to ascertain in future research. Finally, self-reported questionnaires are known to be susceptible to recall bias, however the nature of questions included in the instrument aimed to minimise this effect.

\section{Conclusion}

292 Physiotherapists in ROI frequently prescribe ACTs for the management of patients with AECOPD and perceive physical activity to be the most effective individual technique despite a lack of evidence to support its rationale for this purpose. Awareness of national $\mathrm{ROI}$ guidelines for the management of COPD was very low and further investigation is warranted to better understand the reasons for this. 
1. Irish Thoracic Society (ITS), Respiratory Health of the Nation. Ireland; 2018.

2. Global Initiative for Chronic Obstructive Pulmonary Disease (GOLD): Global strategy for the diagnosis, treatment, management and prevention of chronic obstructive pulmonary disease. USA; 2019.

3. Health Services Executive (HSE), Ireland, National Clinical Programme COPD. End to end model of care for COPD. 2019. Available from: https://www.hse.ie/eng/about/who/cspd/ncps/copd/ [Accessed 29 $9^{\text {th }}$ April 2019] [ Accessed 22 ${ }^{\text {nd }}$ September 2020]

4. National Institute for Health and Care Excellence (NICE), Chronic Obstructive Pulmonary Disease in over 16's: diagnosis and management. 2019. Available from: https://www.nice.org.uk/guidance/ng115

[Accessed 2nd February 2020]

5. Burgel PR, Nesme-Meyer P, Chanez P, Caillaud D, Carre P, Perez T, Roche N. Cough and sputum production are associated with frequent exacerbations and hospitalizations in COPD subjects. Chest. 2009;135(4): 975-962.

6. Munkholm M, Mortensen J. Mucociliary clearance: pathophysiological aspects. Clin Physiol Funct Imaging. 2014; 34: 171-177.

7. Reychler G, Debier E, Contal O, Audag N. Intrapulmonary percussive ventilation as an airway clearance technique in subjects with chronic obstructive airway diseases. Respir Care. 2018; 63: 620- 631 
8. Mcllwaine M, Bradley J, Elborn S, Fidelma M. Personalising airway clearance in chronic lung disease. Eur Respir Rev. 2017; 26:1-12

9. Main E, Denehy L. Cardiorespiratory Physiotherapy $5^{\text {th }}$ Edition. United Kingdom: Elsevier; 2016.

10. Osadnik C, Stuart-Andrews C, Ellis S, Thompson B, McDonald C, Holland A. Positive expiratory pressure via mask does not improve ventilation inhomogenity more than huffing and coughing in individuals with stable chronic obstructive pulmonary disease and chronic sputum expectoration. Respiration. 2014;87(38-44): 38-44.

11. Daynes E, Jones A, Greening N, Singh S. The Use of Airway Clearance Devices in the Management of Chronic Obstructive Pulmonary Disease: A Systematic Review and Meta-Analysis of Randomized Controlled Trials. Ann Am Thorac Soc. 2020

12. Bellone, A., Spagnolatti, L., Massobrio, M., Bellei, E., Vinciguerra, R., Barbieri, A. Short term effects of expiration under positive pressure in patients with acute exacerbation of chronic obstructive pulmonary disease and mild acidosis requiring non-invasive positive pressure ventilation. Intensive Care Medicine. 2002; 28(5):581-585

13. Alghamdi S, Barker R, Alsulayyim A, Alasmari A, Banya W, Polkey M, Birring $\mathrm{S}$, Hopkinson N. Use of oscillatory positive expiratory pressure (OPEP) devices to augment sputum clearance in COPD: a systematic review and meta-analysis. Thorax 2020; 75:855-863 
14. Osadnik CR, McDonald CF, Holland AE. Airway clearance techniques for chronic obstructive pulmonary disease. Cochrane Database of Systematic Reviews. 2012, Issue 3. DOI: 10.1002/14651858.CD008328.pub.2

15. Yohannes AM, Connolly MJ. A national survey: percussion, vibration, shaking and active cycle of breathing techniques used in patients with acute exacerbations of chronic obstructive pulmonary disease. Physiotherapy. 2007; $93: 110-113$

16. Harth L, Stuart J, Montgomery C, Pintier K, Czyzo S, Hill, K et al. Physical therapy practice patterns in acute exacerbations of chronic obstructive pulmonary disease. Can Resp J. 2009; 16(3): 86 - 92

17. Jingar A, Krishna A, Vaishali K, Shyam K, Zulfeequer, Unnikrishnan B. Clinical management practices adopted by physiotherapists in India for chronic obstructive pulmonary disease: A national survey. Lung India. 2013; 30(2):131- 138

18. Osadnik CR, McDonald, CF, Holland AE. Airway clearance techniques in acute exacerbations of COPD: a survey of Australian physiotherapy practice. Physiotherapy. 2013;99: 101-106

19. Westerdahl E, Osadnik, C, Emtner M. Airway clearance techniques for patients with acute exacerbations of chronic obstructive pulmonary disease; Physical therapy practice in Sweden. Chronic respiratory disease. 2019;16:18

20. Australian Institute of Health and Welfare website. Chronic Obstructive Pulmonary Disease. 2018. Available from: 
[Accessed 2th April 2019]

21. Johansson G, Mushnikov V, Backstrom T, Engstrom A, Kahlid J, Wall J. et al. Exacerbations and healthcare resource utilization among COPD patients in a Swedish registry-based nation-wide study. BMC Pulmonary Medicine. 2018;

18:7

22. Hill K., Patman S, Brooks D. Effect of airway clearance techniques in patients experiencing an acute exacerbation of chronic obstructive pulmonary disease: A systematic review. Chronic Respiratory Disease. 2015; 7(1):9-17

23. Brown P, Manfreda J, McCarthy D. The effect of mechanical vibration in patients with acute exacerbations of chronic obstructive pulmonary disease. Can Physiotherapy. 1987; 39(37): 371-374

24. Dwyer T, Alison J, McKeough Z, Daviskas E, Bye P. Effects of exercise on respiratory flow and sputum properties in patients with cystic fibrosis. Chest . $2011 ; 139(4): 870-877$

25. Dwyer T, Zainuldin R, Daviskas E, Bye, P, Alison, J. Effects of treadmill exercise versus Flutter on respiratory flow and sputum properties in adults with cystic fibrosis: a randomised, controlled, cross-over trial. BMC Pulmonary Medicine. 2017;17(14)

26. DeGaris J, Osadnik C. Physical exercise during acute exacerbations of chronic obstructive pulmonary disease: Australian physiotherapy practice. Chronic Respiratory Disease. 2020; 171(8) 
27. Alison J, McKeogh, Z, Johnston K, McNamara R, Spencer L, Jenkins S, Hill C, McDonald V, Frith P, Cafarella P, Brooke M, Cameron-Ticker H, Candy S, Cecins N, Chan A, Dale M, Dowman L, Granger C, Halloran S, Jung P, Lee A, Leung R, Matulick T, Osadnik C, Roberts M, Walsh J, Wootton S, Holland A. Australian and New Zealand Pulmonary Rehabilitation Guidelines. Respirology. 2017; 22, 800-819

28. Kjaergaard, J, Carsten, B, Lange, P, Wilcke, J. Early pulmonary rehabilitation after acute exacerbation of COPD: a randomised controlled trial. ERJ Open Research. 2020; 629. British Thoracic Society (BTS). BTS Quality Standards for Pulmonary Rehabilitation Adults; 2018

\section{.}

8

9

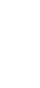

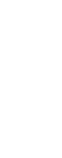

(

(1)




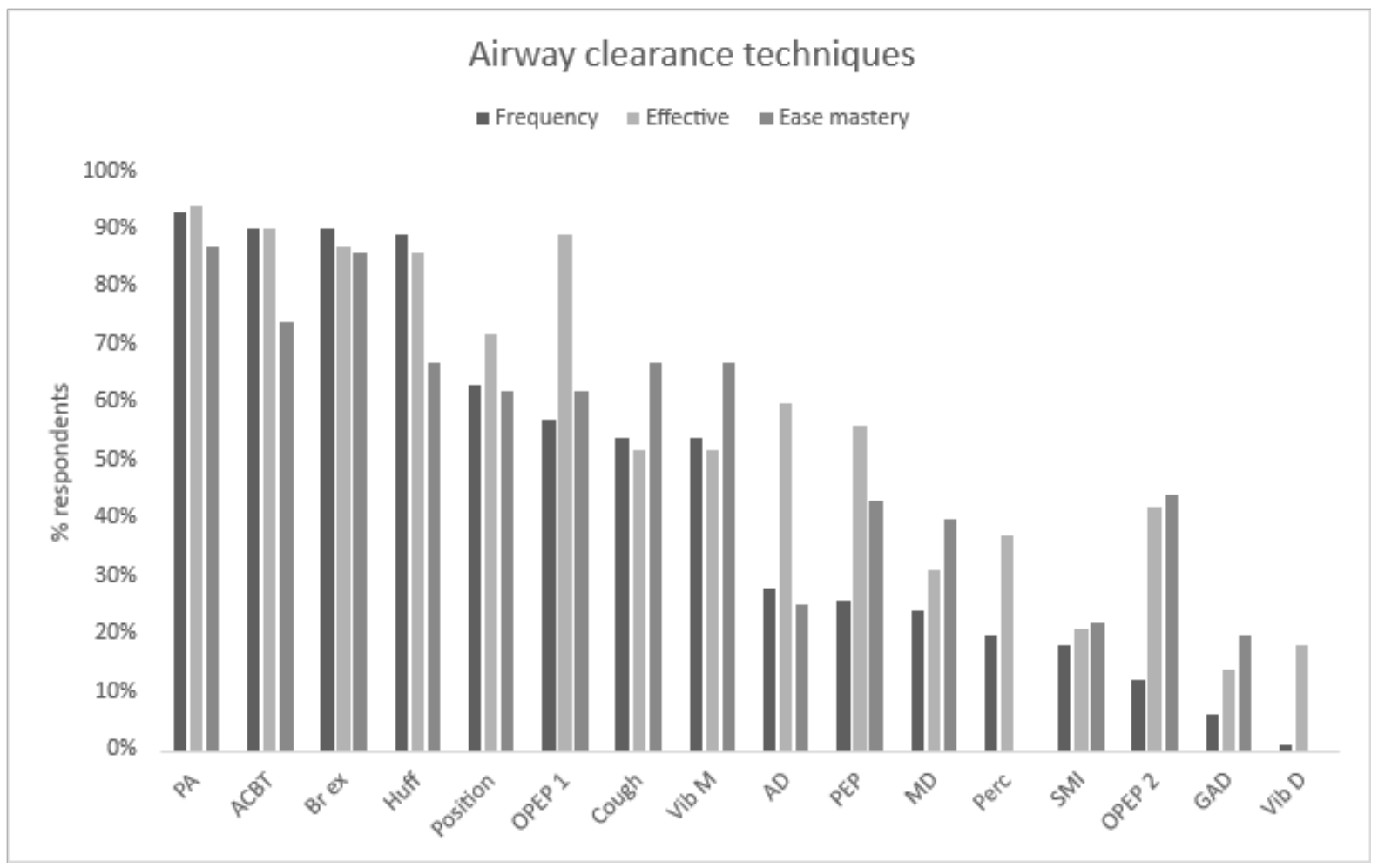

421 Figure 1. Frequency of use, perceived effectiveness and perceived ease of patients' mastery of airway clearance techniques by physiotherapists in the Republic of Ireland. For each of frequency, effectiveness and mastery, Likert options were combined as follows; Frequency; very often or always/often. Effectiveness; very effective/ effective. Mastery; very easy/easy

$\mathrm{PA}=$ physical activity; $\mathrm{ACBT}=$ active cycle of breathing techniques; Huff= huffing; $\mathrm{Br}$ ex= breathing exercise; Position = positioning; OPEP 1= oscillating PEP device (Flutter, Acapella, Cornet); Cough= coughing; Vib $M=$ manual vibration; $A D=$ autogenic drainage; $P E P=$ positive expiratory pressure; $M D=$ manual drainage; Perc= percussions; SMI= sustained maximal inspiration; OPEP $2=$ oscillating PEP (bubble/bottle); GAD= gravity assisted drainage; Vib $\mathrm{D}=$ mechanical vibration via device 


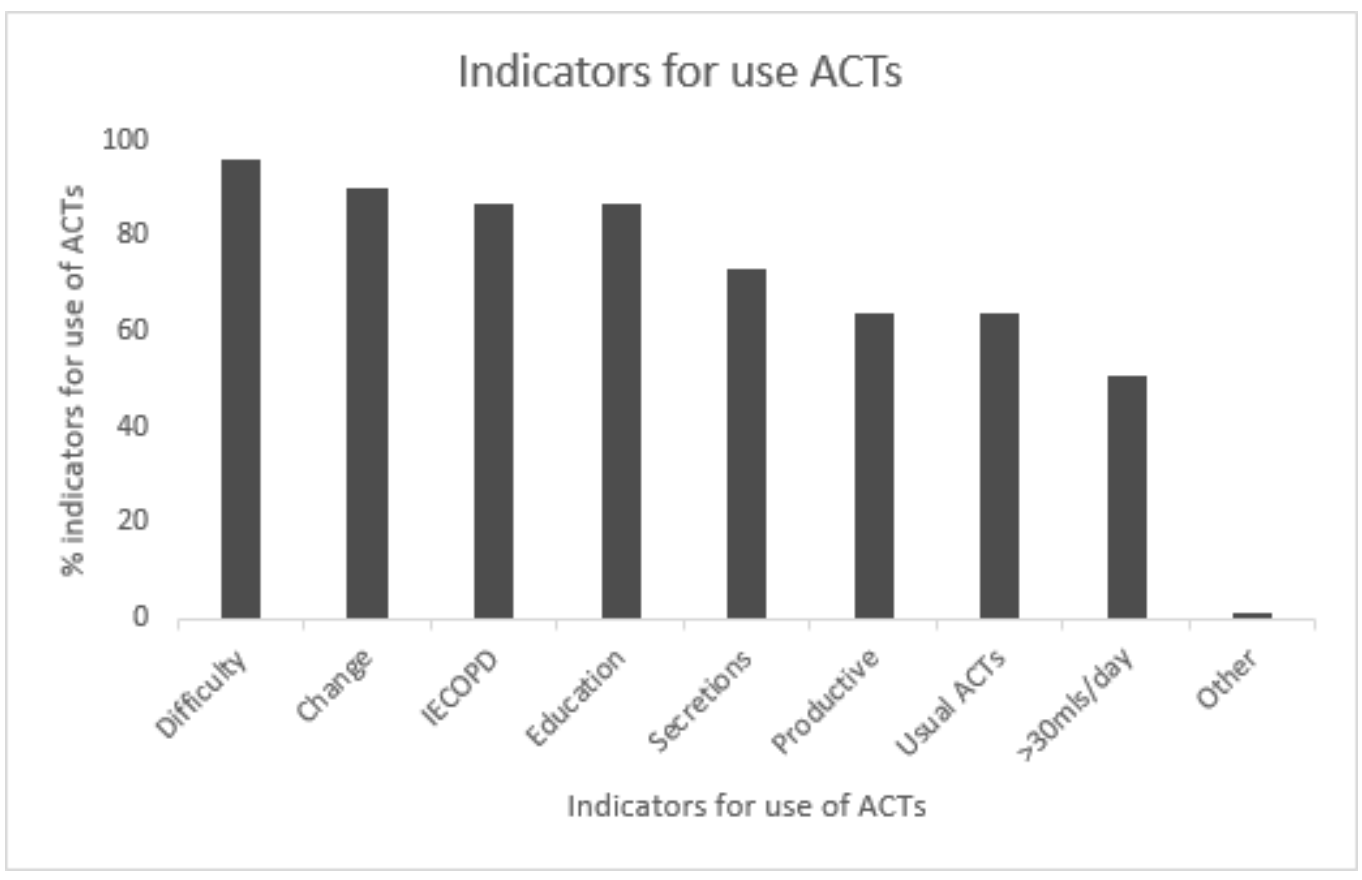

437 Figure 2. Indicators for use of ACTs by physiotherapists in the Republic of Ireland.

438 Difficulty= difficulty managing secretions; Change= a recent change in sputum characteristics;

439 IECOPD= clinical signs suggestive of infectious exacerbation; Education= patient in need of 440 education; Secretions- presence of secretions; Productive= anyone with productive cough; Usual

441 ACTs= patients who usually perform ACTs as part of management of their condition; $>30 \mathrm{mls} /$ day= 442 patients who produce $>30 \mathrm{mls}$ sputum/day; Other. 